\title{
Optimizing Genetic Algorithm Performance for Effective Traffic Lights Control using Balancing Technique (GABT)
}

\author{
Mahmoud Zaki Iskandarani \\ Faculty of Engineering \\ Al-Ahliyya Amman University, Amman, Jordan
}

\begin{abstract}
Genetic Algorithm (GA) is implemented and simulation tested for the purpose of adaptable traffic lights management at four roads-intersection. The employed GA uses hybrid Boltzmann Selection (BS) and Roulette Wheel Selection techniques (BS-RWS). Selection Pressure (SP) and Population (Pop) parameters are used to tune and balance the designed GA to obtain optimized and correct control of passing vehicles. A very successful implementation of such parameters resulted in obtaining minimum number of Iterations (IRN) for a wide spectrum of SP and Pop. The algorithm is mathematically modeled and analyzed and a proof is obtained regarding the condition for balanced GA. Such Balanced GA is most useful in traffic management for an optimized Intelligent Transportation Systems, as it requires minimum iterations for convergence with faster dynamic controlling time.
\end{abstract}

Keywords-Genetic algorithm; traffic lights; intelligent transportation systems; correlation; roulette wheel selection; boltzmann selection; selection pressure; population

\section{INTRODUCTION}

As a result of population growth, increased level of pollution, people migration to urban areas from rural and to cities from urban areas, traffic congestion issue became more critical and pressing. Hence, Traffic lights optimization using adaptive and intelligent algorithms in conjunction with smart sensing is a must under these circumstances. This comes under design optimization, where smart algorithms such as genetic and fuzzy logic algorithms are employed to improve an existing transportation network as a function of increasing level of traffic flow levels, resulting in traffic congestion, delays, higher fuel consumption, air pollution and increase probability of traffic incidents [1-2].

In conjunction with population growth, a marked increase in the automotive industry is witnessed, where millions of vehicles are put on an existing roads. Monitoring the vehicular activities is an important issue to the transport authorities. Such large number of vehicles have a major impact on the environment and daily living of people using these roads due to congestion and delays [3-4].

The congestion issue forms a bigger problem on lanes and roads that are not structured appropriately, where it has a major effect on vehicular movements in a city. In particular at the intersections or with every region of traffic signal, especially during the peak hours. Shortening the duration of the traffic light signal, would not greatly improve traffic flow as important parameters, such as, que length and vehicle speed are not taken into account in a standard control algorithm [5].

Traffic congestion is a critical issue in affecting the lives of the society. Many areas suffered from long term socioeconomic damage owing to growing traffic congestion. To resolve urban congestion, conventional alternatives of increasing road capacity through road network expansion is limited in effectively reducing congestion, as large capital investment and stakeholders support is needed.

Intelligent Transport System (ITS) is used for the last few years in an effort to enhance efficiency and effectiveness of the existing transportation infrastructure by employing various sensor and communication technologies. Adaptive traffic signal control systems try to use the principles provided by artificial intelligence in an attempt to reduce congestion and provide safer traffic operations for both vehicles and pedestrians.

\section{BACKGROUND}

Excessive Traffic density on the roads is a critical issue, as it leads to congestion. This is caused by rise in the number of vehicles and due to expansion and urbanization. Limitations on development and building of new highways and roads, initiated the need to optimize the use of existing infrastructure to achieve optimal flow of traffic. In addition, important time wasted because of traffic congestion, will implicitly affect productivity and performance, and thus affects people's lives, both economically and socially.

Traffic light signal management and control has a marked impact on the efficiency and effectiveness of urban transportation systems. Conventional Traffic light signals are pre-programmed, pre-timed signals. Pre-timed control comprises a series of fixed duration intervals that are repeated continuously. Advanced Traffic light signals can operate in two ways:

1) Actuated mode: Actuated Traffic Light Signals detect and respond to the presence of vehicles or pedestrians at the intersection. They are supported by detectors within the intersection and the necessary control functionality to respond to traffic density and demand, in order to affect the signal cycles times dynamically. 
2) Adaptive mode: Adaptive Traffic Light Signals control system continuously calculates optimal signal timings based on detected volumes and dynamically implement them. The system smartly and efficiently responds to the abrupt and fast changes in dynamic traffic conditions. It uses data from vehicle detectors so that traffic signal optimization is achieved.

Transportation network optimization (TNO) applied to traffic signal control, is implemented while considering route choice pattern of network users. It also include minimization of travel time and avoidance of new road construction. Conventional traffic lights operates using a constant switching cycle regardless of traffic load. Such static mechanism will not allow for variation of traffic load, events, emergency conditions, or general road incidents. Thus, there is a need for smart and adaptive algorithm that dynamically control traffic signals not only locally, but also all over the network for both vehicles and pedestrians. Such a critical solutions will carry out functional synchronization between traffic signals, in order to achieve a measurable reduction in congestion and delay levels, in addition to less pollution and safer driver and pedestrian roads. Such an objective can be achieved using genetic algorithm (GA) [6-10].

Genetic algorithms are used to imitate the processes of natural selection, where the best individuals have more probability to survive and their genes will be part of the creation of one or more offspring. Such process is repeated with the output of each new offspring is more fitted to survive than its parents. In the last few years, genetic algorithms found to present a suitable approach to complex transportation problems, as they are considered search algorithms that operate on the principles of natural selection. They determine a number of potential solutions within a population, with an encoding process that result in an optimized solution. In transportation, genetic algorithms are used to optimize and adapt the green interval response as a function of traffic density based on vehicles count [11-15].

Selecting the best parameters of a genetic algorithm, so as to obtain good results to optimize its performance, is very important to its effectiveness. Crossover, mutation rate and population size are the most influencing control parameters as reported by previous works [16-20]. However, pressure selection and population size in correlation is a new approach in balancing and GA algorithm optimization.

In this paper an investigation is carried out regarding selection pressure and population parameters within a GA algorithm used for intelligent traffic lights control and management, in order to obtain best adaptable performance under dynamically changing traffic density. The two parameters and their correlative effect on the performance of the employed GA algorithm is mathematically analyzed, simulated and results discussed.

\section{METHODOLOGY}

The main objective of this work is to optimize the designed genetic algorithm in order to produce an optimum traffic lights control mechanism, which is adaptable in nature and intelligent in behavior.

Selection process is a critical part in genetic algorithms, whereby chromosome is chosen from the available generation's population to be included in the next generation. Fitness function based process selects the best chromosomes, which is used to improve chances of individual survival.

The employed probability of selection process is based on two known algorithms:

1) Boltzmann Selection (BS): The algorithm is based on simulated annealing. Annealing is based on cooling such that a low energy state is reached. During the process, heating until melting is reached at a high temperature, through which, random movement is realized. The temperature is slowly cooled until the minimum energy states achieved. The equivalence between the optimization process in this work and the Boltzmann Simulated Annealing (SA) is the need to achieve stable levels with better new solutions (optimized traffic signal timing). Boltzmann Selection (BS), general expression is presented in equation (1).

$$
P_{\text {Selection }}\left({ }_{i+1}\right)=\beta \exp \left(-\frac{y_{i+1}-y_{i}}{T_{i+1}}\right) \text {. }
$$

Where;

$\mathrm{y}_{\mathrm{i}}$ : Fitness function value for initial solution

$\mathrm{y}_{\mathrm{i}+1}$ : Fitness function value for the new solution

$\mathrm{T}$ : New artificial temperature

\section{$\beta$ : Normalization factor}

In the algorithm used in this work, the rate of selection is managed by a continuously changing Delay Time (DT) parameter, which is equivalent to temperature in Boltzmann algorithm (simulated annealing). Initially Delay Time (DT) is high. So, one DT (Shortest Delay Time (SDT)) parameter will be at first high and decreases, with another DT (Longest Delay Time (LDT)) providing Delay Time as a function of increase in the testing population. (SDT) decreases gradually which increases the effect of Selection Pressure (SP). This results in determining and mapping the search space. In Boltzmann selection, the probability of selecting best value is high with lower execution time.

2) Roulette Wheel Selection (RWS): In this algorithm, selection of the fittest is carried out. The initial part of the selection process based on stochastic selection from one population to create the basis for the next population. In this process, the fittest have a better chance (probability) of survival than weakest ones. Thus, the fittest will move forward to the mating region to prepare for the next population. The process is shown in Fig. 1. 


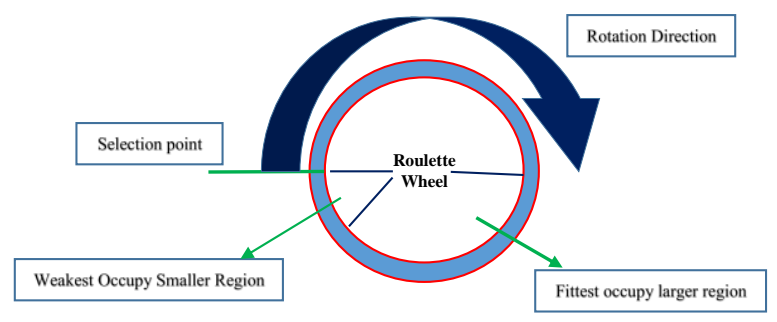

Fig. 1. Roulette Wheel Selection.

The number of times the roulette wheel runs is proportional to the size of the desired population. Whenever the wheel stops fittest individual will have a good chance of being selected for the next population and subsequent mating region.

The algorithm applies the expression in equation (2):

$$
P_{\text {Selection }}\left({ }_{m}\right)=\left(\frac{y_{m}}{\sum_{k=1}^{n} y_{k}}\right)
$$

Where;

\section{n: Population Size}

m: Number of times the wheel rotated

The combined Boltzmann Selection-Roulette-Wheel Selection (BS-RWS), for genetic algorithms (GAs) is based on both entropy and importance sampling methods. It naturally leads to adaptive fitness in which the fitness function does not stay fixed but dynamically varies.

Two important controlling parameters are used in optimizing and validating the used genetic algorithm for a fourtraffic lights intersection covering four roads (Rd.1, Rd.2, Rd.3, Rd.4), each having a capacity of 60 vehicles:

1) Selection Pressure (SP)

2) Population (Pop)

Selection Probability (SProb) for traffic lights control, which in effect manages the number of vehicles passing from road to road at the four-road junction is governed by equation (3), which is based on Boltzmann general expression in equation (1).

$S \operatorname{Pr} o b=\exp \left(\frac{-S P x S D T}{L D T}\right)$.

Where;

(SDT): Shortest Delay Time (Best Time), which is a dynamic parameter that varies with number of iterations (IRN).

(LDT): Longest Delay Time (Worst Time), which is a dynamic parameter that corresponds to Temperature in
Boltzmann Selection (BS) and varies with Population exploration. Equation (3) can be rearranged as in equation (4)

$S \operatorname{Pr} o b=\exp \left(-S P\left(\frac{S D T}{L D T}\right)\right)$.

SDT and LDT are important parameters in the genetic algorithm computations, as they are related to the used fitness function. They are vital for the designed algorithm in determining the value of SProb, and thus, affect its controlling mechanism and number of Passing Vehicles (PV). Hence, SProb is a function of both the Selection Pressure (SP) and the dynamic ratio of the genetic algorithm times that depends on the population value, which is related to other parameters such as offsprings, mutants and crossover levels.

Thus, selection of SP and Pop, will affect SProb, which in turn affects the algorithm computations and Vehicles Passing Rate (VPR). So, balancing and optimization of the two values will result in a tuned, balanced and efficient GA for traffic signals and traffic control and management.

Two conditions associated with Selection Pressure:

1) High Selection Pressure Value: Early convergence.

2) Low Selection Pressure Value: Late convergence.

Thus, an optimum selection pressure value is needed for optimal control of traffic lights, which is a dynamic, changeable value function of traffic density, which is computed in the designed algorithm as in equations (5) and (6) for each road $\mathrm{i}$.

$$
\begin{aligned}
& T P_{i}=\left(R C_{i}-V P_{i}\right) . \\
& T P R_{i}=\left(\frac{\left(R C_{i}-P V_{i}\right)}{R C_{i}}\right) .
\end{aligned}
$$

Where;

TP: Traffic Pressure

TPR: Traffic Pressure Ratio

PV: Passing Vehicles

RC: Road Capacity

$\mathrm{i}$ : Road at the 4-road intersection $(\mathrm{i}=1,2,3,4)$

PV is related to both SP and Pop, subsequently is affected by SProb, which is a function of SDT and LDT and SP. Equal PV for same TP per road should be equal for the GA algorithm to be considered balanced and optimized. The general process of optimization is shown in Fig. 2.

The idea is to optimize the GA performance using both SP and Pop to match the balancing data shown in Table I. 


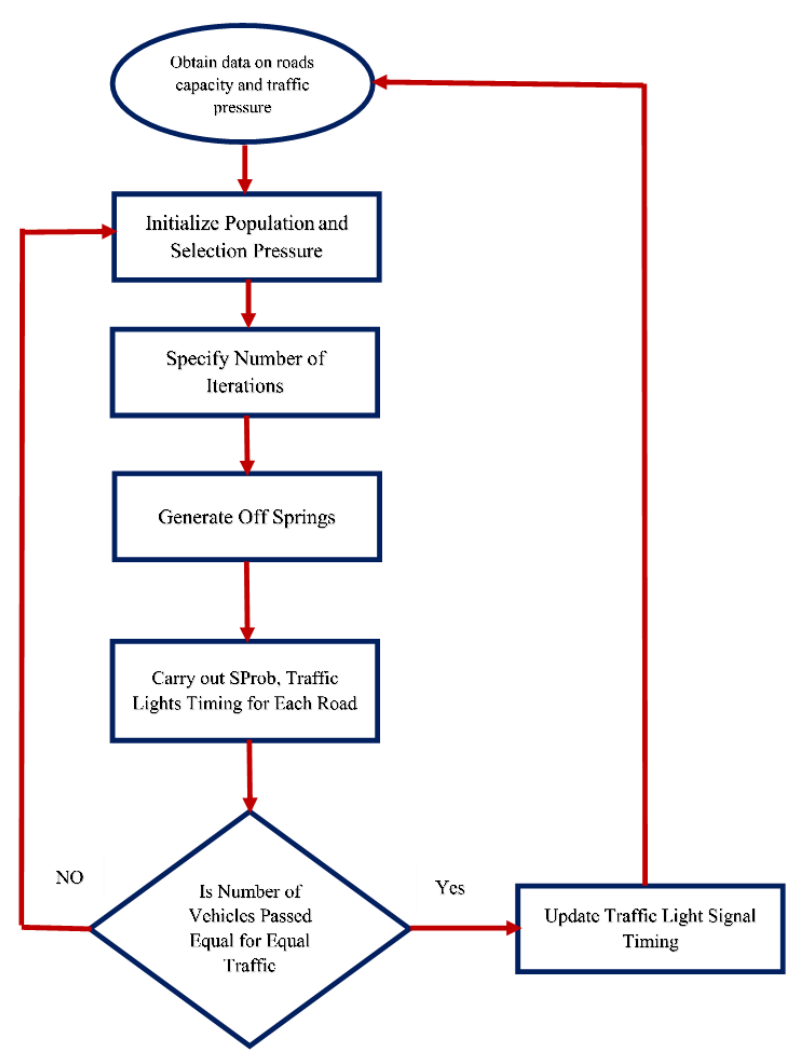

Fig. 2. GA Optimization Algorithm.

TABLE. I. GA BALANCING REFERENCE VALUES

\begin{tabular}{|l|l|l|l|l|l|l|l|l|}
\hline \multirow{2}{*}{ POP $_{i}$} & \multicolumn{3}{|c|}{ Vehicles Passing Times (sec) } & \multicolumn{3}{c|}{ No. of Passing Vehicles } \\
\cline { 2 - 9 } & Rd.1 & Rd.2 & Rd.3 & Rd.4 & Rd.1 & Rd.2 & Rd.3 & Rd.4 \\
\hline SP & & & & & & & & \\
\hline $\mathbf{2}$ & 17 & 57 & 57 & 17 & 7 & 23 & 23 & 7 \\
\hline $\mathbf{4}$ & 17 & 57 & 57 & 17 & 7 & 23 & 23 & 7 \\
\hline $\mathbf{6}$ & 17 & 57 & 57 & 17 & 7 & 23 & 23 & 7 \\
\hline $\mathbf{8}$ & 17 & 57 & 57 & 17 & 7 & 23 & 23 & 7 \\
\hline $\mathbf{1 0}$ & 17 & 57 & 57 & 17 & 7 & 23 & 23 & 7 \\
\hline
\end{tabular}

\section{RESULTS}

Table II shows the number of Iterations (IRN) required for each Selection Pressure Value to achieve balanced and optimized GA performance as a function of the relationship between Population (Pop) and Selection Pressure (SP), with plots representing the data shown in Fig. 3 as a function of SP and in Fig. 4 as a function of Pop.

TABLE. II. IRN AS A FUNCTION OF BOTH SP AND POP

\begin{tabular}{|l|l|l|l|l|l|}
\hline Iterations (IRN) & \multicolumn{5}{|l|}{ Population (Pop) } \\
\hline SP & 200 & 400 & 600 & 800 & 1000 \\
\hline $\mathbf{2}$ & 650 & 350 & 300 & 50 & 50 \\
\hline $\mathbf{4}$ & 500 & 200 & 50 & 150 & 50 \\
\hline $\mathbf{6}$ & 300 & 50 & 200 & 50 & 50 \\
\hline $\mathbf{8}$ & 450 & 50 & 200 & 50 & 50 \\
\hline $\mathbf{1 0}$ & 950 & 400 & 400 & 400 & 50 \\
\hline
\end{tabular}

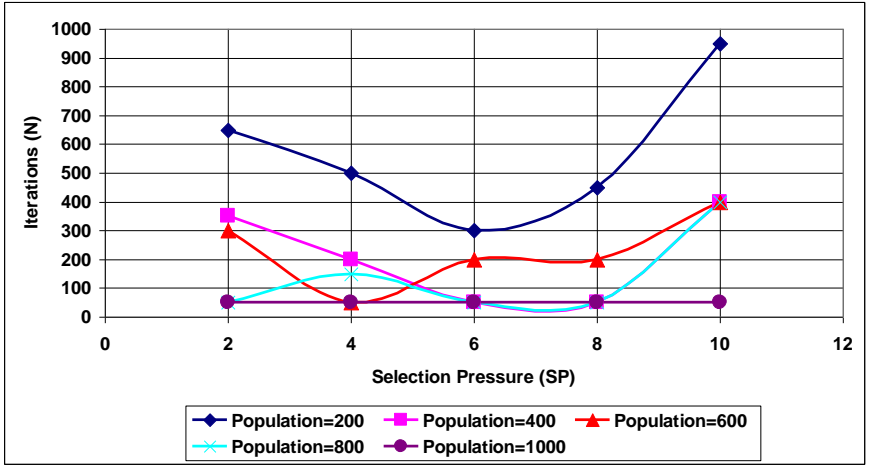

Fig. 3. Iterations (IRN) as a Function of SP Per Specific Pop.

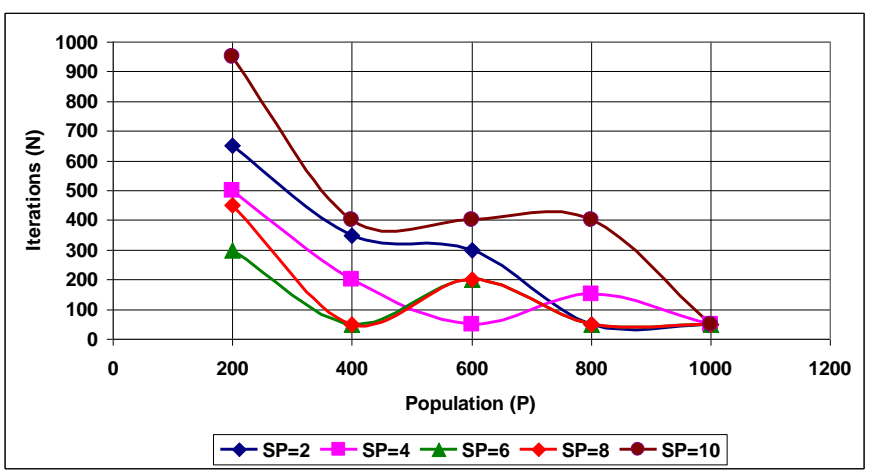

Fig. 4. Iterations (IRN) as a Function of Pop Per Specific SP.

\section{ANALYSIS AND DISCUSSION}

The analysis process is based on the stability criteria, whereby, a correlation between three parameters (SP, Pop, Iterations) is carried out, with final correlation to smooth and correct traffic lights control at the junction, such that the four roads will pass the correct number of vehicles. This is a calibration and load balancing measure, which is based on initial conditions of equal traffic pressure for opposing roads.

Table II shows data represent the Iteration parameter as a function of the relationship between SP and Pop, whereby Iterations are dependent on the two parameters (SP, Pop) with each SP level is fixed for a variable levels of Pop. The general form of dependency is described by equation (7).

$I R N=f(S P, P o p)$.

Now, Pop affects LDT, hence affecting SProb. Also, SP affects SProb according to equation (4). Thus, IRN, will affect SProb and by using both equations (4) and (7), equation (8) is obtained.

$S \operatorname{Pr} o b=f(I R N)$.

The PV parameter depends on SProb, hence, can be described by equation (9).

$P V=g(S \operatorname{Pr} o b)=g(f(I R N))$. 
Then, for each opposite and equal TP roads, the difference in the rate of change should be zero for balanced GA algorithm. Thus; for i (1 to 4$)$ :

$\Delta P V_{i}=\Delta g_{i}\left(f\left(I R N_{i}\right)\right)=0$.

The condition in equation (10) will only apply if the number of iterations in the GA algorithm is the same for each SP and Pop to enable same number of vehicles to pass on each road as a function of TP. This condition is valid for stable GA algorithm. Hence, when achieved, the GA algorithm is balanced and optimized as equation (11) shows.

$\Delta I R N_{i}=0$.

From Table II, it is clear that the Steady State (SS) values is reached for the GA algorithm controlling four traffic lights with one intersection at $\mathrm{Pop}=1000$, with minimum number of Iterations of 50, which is also constant for all levels of SP. Such convergence, appears in Fig. 2.

Table III, presents data that shows effect of Pop on IRN in order to reach SS, with Fig. 3 showing the convergence to an $\mathrm{SS}$ described by the pairing in equation (12):

$S S=(P o p, I R N)$.

From Table II, it is realized that for some SP, Pop values oscillation occurs during tuning and balancing of the GA algorithm. The two non-oscillatory values are shown in Table III, with $\mathrm{SP}=2$ having early convergence to minimum number of iterations. Non-oscilatory SP, Pop pairs are prefered in the balancing process to other values, eventhough the convergence condition achived with intermediate GA oscillations.

Fig. 5 to 8 show SDT and LDT for the two non-oscillatory SP values.

TABLE. III. NON-OSCILLATORY SP AND POP

\begin{tabular}{|l|l|l|l|l|l|}
\hline Iterations (IRN) & \multicolumn{5}{|l|}{ Population (Pop) } \\
\hline SP & 200 & 400 & 600 & $\mathbf{8 0 0}$ & 1000 \\
\hline 2 & 650 & 350 & 300 & 50 & 50 \\
\hline 10 & 950 & 400 & 400 & 400 & 50 \\
\hline
\end{tabular}

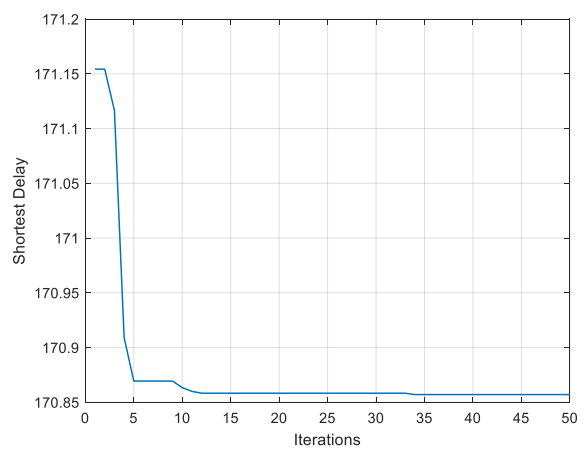

Fig. 5. $\{2,1000\}$ SDT Curve.

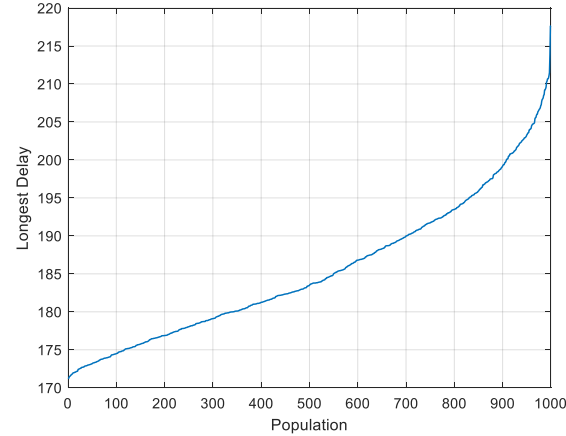

Fig. 6. $\{2,1000\}$ LDT Curve.

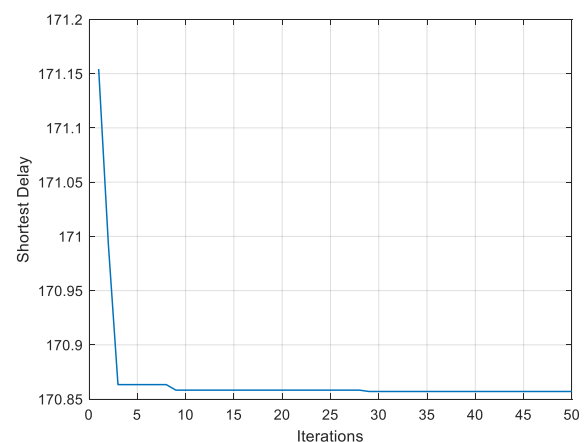

Fig. 7. $\{10,1000\}$ LDT Curve.

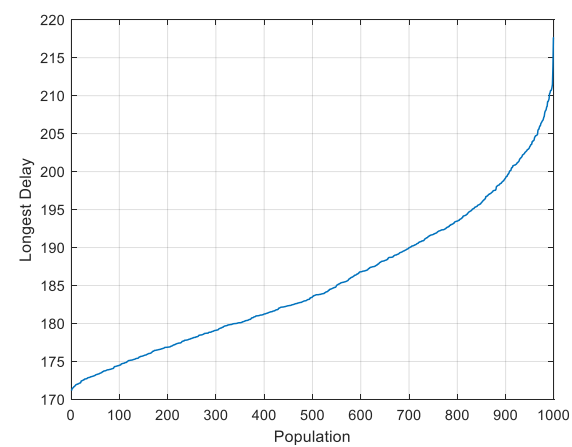

Fig. 8. $\quad\{10,1000\}$ LDT Curve.

\section{CONCLUSIONS}

The obtained condition for number of interactions (IRN) in this work and subsequent steady state condition specified, is an important achievement, as it uncovers conditions of constant and low number of iterations necessary to obtain balanced, and optimized GA control algorithm used in the management of traffic lights signaling process. The used technique locally weights the processes using both Pop and SP correlation, which enables optimized functionality and better forecasts to achieve optimal dynamic traffic modeling. The balanced GA algorithm proposed in this work offers tangible advantages. Future work requires the extension of such application of balanced GA algorithm is recommended to cover multi-section traffic control, whereby each four-road, single intersection is regarded as a single parameter and correlated with other similar structure to cover more complex arrangement. 


\section{REFERENCES}

[1] J. Chen, Y. Yu, Q. Guo, "Freeway Traffic Congestion Reduction and Environment Regulation via Model Predictive Control," Algorithms, vol. 12, no. 220, pp. 1-23, 2019.

[2] G. Jia , R. Ma, Z. Hu , "Review of Urban Transportation Network Design Problems Based on CiteSpace," Journal of Theoretical and Applied Information Technology, vol. 2019, ID. 5735702, pp. 1-22, 2019.

[3] A. Goyal, M. Singh, A. Aeron, "Simulation of Traffic Optimization to Reduce Congestion," International Journal of Innovative Technology and Exploring Engineering, vol. 8, no. 11, pp. 3780-3783, 2019.

[4] S. Yang, Y. Ji, D. Zhang, J. Fu, "Equilibrium between Road Traffic Congestion and Low-Carbon Economy: A Case Study from Beijing, China," sustainability, vol. 2019, no. 11, pp. 1-22, 2019.

[5] B. Sony, A. Chakravarti, M. Reddy, "Traffic Congestion Detection using Whale Optimization Algorithm and Multi Support Vector Machine," Journal of Theoretical and Applied Information Technology, vol. 7, no. 6C2, pp. 589-593, 2019.

[6] Q. Bing , D. Qu , X. Chen , F. Pan, J. Wei, "Short-Term Traffic Flow Forecasting Method Based on LSSVM Model Optimized by GA-PSO Hybrid Algorithm," Discrete Dynamics in Nature and Society, vol. 2018, ID. 3093596, pp. 1-10, 2018.

[7] Y. Chen L. Rilett, "Signal Timing Optimization for Corridors with Multiple Highway-Rail Grade Crossings Using Genetic Algorithm," Journal of Advanced Transportation, vol. 2018, ID. 9610430, pp. 1-14, 2018.

[8] N. Saharkar , M. Wanjari, "A Genetic Algorithm Based Approach to Solve Transport Problems for School Buses?" Journal of Engineering and Applied Sciences, vol. 13, no. 4, pp. 848-851, 2018.

[9] T. Karthy and K .Ganesan, "Multi Objective Transportation Problem Genetic Algorithm Approach," International Journal of Pure and Applied Mathematics, vol. 119, no. 9, pp. 343-350, 2018.

[10] E. Han, H. PiLee, S. Park, J. So, I. Yun, "Optimal Signal Control Algorithm for Signalized Intersections under a V2I Communication Environment," Atmoshphere, vol. 2019, ID. 6039741, pp. 1-9, 2019.
[11] A. Potnurwar, S. Aote, V. Bongirwar, "Design of Traffic Volume Forecasting based on Genetic Algorithm," International Journal of Recent Technology and Engineering, vol. 8, no. 2, pp. 4264-4268, 2019.

[12] A. EL Idrissi, C. Tajani, M. Sabbane, "New Crossover Operator for Genetic Algorithm to Resolve The Fixed Charge Transportation Problem," Journal of Theoretical and Applied Information Technology, vol. 98, no. 8, pp. 1607-1617, 2017.

[13] W. Wen-jing, "Improved Adaptive Genetic Algorithm for Course Scheduling in Colleges and Universities," iJET, vol. 13, pp. 29-42, 2018.

[14] Y. Wang, X. Yang, H. Liang, Y. Liu, "A Review of the Self-Adaptive Traffic Signal Control System Based on Future Traffic Environment," Journal of Advanced Transportation, vol. 2018, ID. 1096123, pp. 1-12, 2018.

[15] Q. Tang, B. Friedrich, "Design of Signal Timing Plan for Urban Signalized Networks including Left Turn Prohibition," Journal of Advanced Transportation, vol. 2018, ID.1645475, pp. 1-16, 2018.

[16] S. Srivastava, S. Sahana, "Application of Bat Algorithm for Transport Network Design Problem," Applied Computational Intelligence and Soft Computing, vol. 2019, ID. 9864090, pp. 1-12, 2019.

[17] Z. Cakici, Y. Sazi Murat, "A Differential Evolution Algorithm-Based Traffic Control Model for Signalized IntersectionsAdvances in Civil Engineering, vol. 2019, ID. 7360939, pp. 1-16, 2019.

[18] C. Canali, R. Lancellotti, "GASP: Genetic Algorithms for Service Placement in Fog Computing Systems," Algorithms, vol. 12, pp. 1-19, 2019.

[19] A. Rahman, N. Shahruddin, Ismail Ishak, "Solving the Goods Transportation Problem Using Genetic Algorithm with Nearest-Node Pairing Crossover Operator, " Journal of Physics: Conference Series, vol. 1366, pp. 1-9, 2019.

[20] X. Feng, X. Zhu, X. Qian, Y. Jie, F. Ma, and X. Niu, "A new transit network design study in consideration of transfer time composition," Transportation Research Part D: Transport and Environment, vol. 66, pp. 85-94, 2019. 